\title{
Bloodstream Infections among Solid Organ Transplant Recipients: Eight Years' Experience from a Turkish University Hospital
}

\author{
Ayşegül Yeşilkaya', Özlem Kurt Azap ${ }^{1}$, Melike Hamiyet Demirkaya ${ }^{1}$, Mehtap Akçıl Ok², Hande Arslan ${ }^{1}$, Aydıncan Akdur ${ }^{3}$ \\ ${ }^{1}$ Department of Infectious Diseases and Clinical Microbiology, Başkent University Faculty of Medicine, Ankara, Turkey \\ ${ }^{2}$ Department of Statistics and Computer Science, Başkent University Faculty of Science and Letters, Ankara, Turkey \\ ${ }^{3}$ Department of General Surgery, Başkent University Faculty of Medicine, Ankara, Turkey
}

\section{ABSTRACT}

Background: Bloodstream infections (BSI) are frequently seen after solid organ transplantation. The incidence of bloodstream infections differs among the types of transplantation. The microbiological features and antimicrobial resistance patterns change from centre to centre.

Aims: To evaluate the incidence and spectrum of aetiological agents of bloodstream infections among solid organ transplantation recipients.

Study Design: Retrospective descriptive study.

Methods: Medical records of solid organ transplant recipients in the period between January $1^{\text {st }} 2004$ and August $15^{\text {th }} 2012$ were assessed retrospectively. The study population comprised 927 (64 heart, 556 kidney, 307 liver) consecutive recipients. Bloodstream infections were divided into three groups according to the onset time of bloodstream infections after transplantation: early, mid-term and late. The incidence and microbiological features of bloodstream infections were evaluated.

Results: The number of bloodstream infection episodes was 317 in 191 recipients which was distributed as $228(72 \%)$ in liver, 70 (22\%) in kidney and 19 (6\%) in heart transplantation. Ninety-eight 98 (30.9\%) of the episodes were diagnosed within the early period, $134(42.3 \%)$ within the mid-term and 85 (26.8\%) in the late period. Early and mid-term bloodstream infections were seen statistically more often in liver than in kidney or heart transplantation ( $p=0.01$ and $p=0.031$, respectively). Late bloodstream infections were also common in liver transplant recipients which was not statistically significant ( $p=0.229$ ).

Conclusion: Liver transplant recipients are at the highest risk for developing BSI after transplantation in early, mid-term and late periods.

Key Words: Bloodstream infection, organ transplantation, Turkey

Received: 07.04.2013

Accepted: 08.05.2013

\section{Introduction}

Bloodstream infections (BSI) continue to be the most important cause of morbidity and mortality among solid organ transplantation (SOT) recipients (1). The recipients of liver transplantation had a higher incidence of BSI compared to the recipients of either kidney or heart transplantation $(2,3)$. The aetiology of bacteraemia in SOT recipients has changed decade by decade. Gram negative bacteria were predominant pathogens in BSI in SOT recipients in the 1980s, whereas gram positive bacteria were in the 1990s. There has again been a shift toward gram negative bacteria in the last decade (2-6).

Our primary aim was to evaluate both the aetiology and incidence of BSI by time period following SOT recipients at a university hospital from 2004 to 2012 in Turkey. The second aim was to evaluate the in vitro antimicrobial susceptibilities of causative agents of bacteraemia throughout the study period.

\section{Material and Methods}

The conducted study was a retrospective descriptive study in which data were collected from medical records for each SOT recipient regarding their age from January $1^{\text {st }} 2004$ to August $15^{\text {th }} 2012$ at a 288 -bed university hospital. Kidney, liver and heart transplants have been performed in our institution since 1985, 1988 and 2003, respectively.

\section{Definition}

BSI were analysed according to the time of onset after solid organ transplantation. We compared the incidence of BSI in SOT recipients within the first month, between two and twelve months and more than one year following the transplantation. We did not analyse incidence rate, risk factors, mortality, outcome and post-transplant complications. The primary source of bacteraemia was not defined.

\section{Blood Cultures}

Blood culture bottles were incubated in the automated continuous monitoring BD BACTEC ${ }^{\text {TM }} 9240$ (Becton Dickinson, Sparks, USA) blood culture system. The identification of organisms was performed using conventional methods and automated systems. Antimicrobial susceptibility testing was done according to the criteria of the Clinical and Laboratory Standards Institute. 


\section{Antibiotic prophylaxis}

The perioperative prophylaxis protocol consisted of cefazolin for two days both for kidney and heart transplantation in our centre. Liver transplant recipients received cefotaxime and ampicillin for three days perioperatively. The recipients also received trimethoprim-sulphamethoxazole for Pneumocystis jirovecii prophylaxis and valganciclovir for cytomegalovirus prophylaxis. Fluconazole prophylaxis was provided for two weeks among liver transplant recipients.

\section{Antibiotic resistance}

Methicillin resistance in Staphylococcus aureus strains, extended-spectrum $\beta$-lactamase (ESBL) production both in $E$. coli and in $K$. pneumoniae strains, and multidrug-resistance (MDR) and extensively drug-resistance (XDR) in Acinetobacter baumannii and Pseudomonas aeruginosa strains were evaluated. The interim standard definitions for MDR and XDR were used as appropriate (8). The percentage of ciprofloxacin, ceftriaxone, carbapenem and amikacin resistance in enteric gram negative bacilli were also evaluated.

\section{Statistical Analysis}

All statistical analyses were carried out using the Statistical Package for Social Science version 11.0 (SPSS Inc., Chicago, IL, USA). Pearson chi-square test was used for comparisons of categorical data and a two-sided $p$ value $<0.05$ was considered to be statistically significant.

This study was approved by Başkent University Institutional Review Board (Project no: KA13/65) and supported by the Baskent University Research Fund.

\section{Results}

\section{Patient Characteristics}

Nine hundred and twenty-seven organ transplantations (64 heart, 556 kidney and 307 liver) were performed between January $1^{\text {st }} 2004$ and August $15^{\text {th }} 2012$ (Table 1). Of these patients, 687 underwent living donor organ transplantation (441 kidney and 246 liver) and 240 recipients a deceased donor organ transplantation (115 kidney, 61 liver and 64 heart). One hundred and ninety-one patients developed $317 \mathrm{BSI}$ episodes [228 (72\%) in liver, $70(22 \%)$ in kidney and $19(6 \%)$ in heart transplantation]. Of these 191 patients, 56 were younger than 18 years of age. The frequency of BSI among 56 paediatric recipients was $26.2 \%$ (83/317episodes). The median age of SOT recipients at the time of BSI was 37 years (1-69 years). The male gender $(129 / 191,67.5 \%)$ was prominent in the recipients with BSI.

\section{Incidence of BSI}

BSI occurred in 191 of the 927 patients (20.6\%). The incidence of BSI by type of transplantation is shown in Table 1. The distribution of 191 transplantation recipients according to the type of transplantation were as follows: 121 liver, 58 kidney and 12 heart. Sixty-nine recipients experienced more than one episode of BSI (2-9 episodes) (Table 1). The type of transplantation differs in those 69 recipients, with 55 (79.7\%) for liver, 10 (14.5\%) for kidney and 4 (5.8\%) for heart transplantation. Liver transplantation recipients experienced more BSI episodes than other organ transplantation types $(p=0.001)$.

Table 1. Characteristics of the recipients of a solid organ transplantation with bloodstream infection, according to the type of transplantation

\begin{tabular}{|c|c|c|c|c|}
\hline & Kidney & Liver & Heart & Total \\
\hline Transplants performed & 556 & 307 & 64 & 927 \\
\hline Living donor & 441 & 246 & 0 & 687 \\
\hline Number of BSI episodes & 70 & 228 & 19 & 317 \\
\hline Number of BSI episodes $>1$ & 10 & 55 & 4 & 69 \\
\hline Number of patients with BSI & $\begin{array}{c}58 \\
(10 \%) \\
\left(\mathrm{Cl}_{95} 8.7-11.3\right)\end{array}$ & $\begin{array}{c}121 \\
(39 \%) \\
\left(\mathrm{Cl}_{95} 36-42\right)\end{array}$ & $\begin{array}{c}12 \\
(19 \%) \\
\left(\mathrm{Cl}_{95} 14-24\right)\end{array}$ & $\begin{array}{c}191 \\
(21 \%) \\
\left(\mathrm{Cl}_{95} 19.7-22.3\right)\end{array}$ \\
\hline Ratio BSI episodes/patients & 1.2 & 1.9 & 1.6 & 1.7 \\
\hline Incidence by episodes & $12.5 \%$ & $74.2 \%$ & $29.6 \%$ & $34.1 \%$ \\
\hline Incidence by patients & $10.4 \%$ & $39.4 \%$ & $18.8 \%$ & $20.6 \%$ \\
\hline \multicolumn{5}{|l|}{ Microbiology of BSI } \\
\hline Gram negative & 42 & 115 & 11 & $168(61.1 \%)$ \\
\hline Gram positive & 20 & 75 & 4 & 99 (36\%) \\
\hline \multicolumn{5}{|l|}{ Ratio of gram positive } \\
\hline to gram negative BSI & 0.47 & 0.65 & 0.36 & 0.58 \\
\hline Candidaemia* & 1 & 7 & 0 & $8(2.9 \%)$ \\
\hline Polymicrobial & 7 & 31 & 4 & 42 (13.2\%) \\
\hline
\end{tabular}


Table 2. Monomicrobiological isolates of bloodstream infections by type of transplantation

\begin{tabular}{|c|c|c|c|c|}
\hline Organism & Total & Kidney & Liver & Heart \\
\hline Gram positives & 99 (36\%) & 20 (32\%) & 75 (38\%) & 4 (27\%) \\
\hline CoNS & 32 (12\%) & 10 & 21 & 1 \\
\hline S. aureus & 19 (7\%) & 5 & 14 & - \\
\hline Enterococci & 37 (13\%) & 2 & 33 & 2 \\
\hline Streptococci & $11(4 \%)$ & 3 & 7 & 1 \\
\hline Gram negatives & 168 (61\%) & 42 (67\%) & 115 (58\%) & 11 (73\%) \\
\hline E. coli & 79 (29\%) & 23 & 55 & 1 \\
\hline Klebsiella spp. & $36(13 \%)$ & 9 & 25 & 2 \\
\hline Enterobacter spp. & $8(3 \%)$ & 2 & 6 & - \\
\hline Pseudomonas spp. & $16(6 \%)$ & 3 & 12 & 1 \\
\hline Acinetobacter spp. & 20 (7\%) & 1 & 12 & 7 \\
\hline S. maltophilia & $4(1 \%)$ & 1 & 3 & - \\
\hline Other & $5(2 \%)$ & 3 & 2 & - \\
\hline Candidaemia & $8(3 \%)$ & $1(2 \%)$ & $7(4 \%)$ & - \\
\hline Total & 275 & 63 & 197 & 15 \\
\hline
\end{tabular}

BSI: bloodstream infection; CoNS: coagulase negative staphylococci

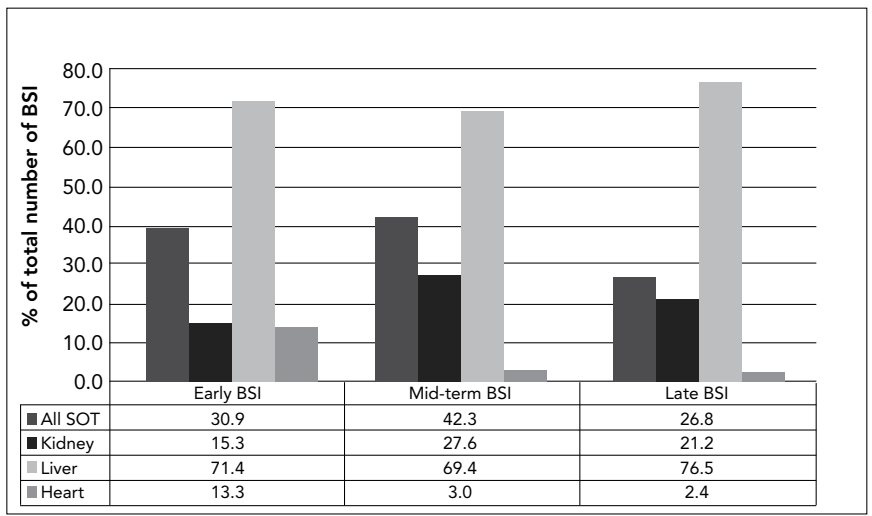

Figure 1. The distribution of bloodstream infections according to the time of onset after transplantation

Ninety-eight (30.9\%) of the total episodes were diagnosed within the first month after transplantation, 134 (42.3\%) within the mid-term and 85 (26.8\%) in the late period (Figure 1). Early BSI was seen more often in liver (71.4\%) than kidney or heart transplantation $(p=0.01)$. Mid-term BSI after liver transplantation $(69.4 \%)$ was also seen more commonly $(p=0.031)$. Late BSI was seen more commonly in liver transplant recipients (76.5\%) compared with other types, but this was not statistically significant $(p=0.229)$ (Figure 1$)$. There was also no statistically significant difference between the time of onset of $B S I$ and pathogen group ( $p=0.535$ for early BSI; $p=0.142$ for mid-term BSI; and $\mathrm{p}=0.336$ for late $\mathrm{BSI}$ ).

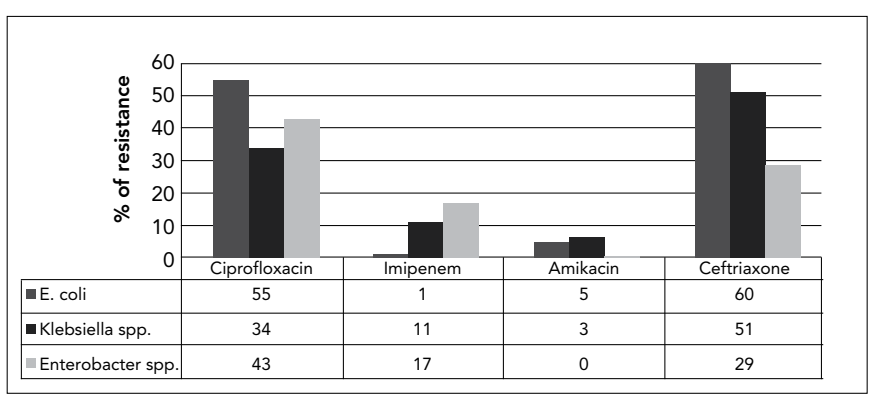

Figure 2. Antibiotic resistance among three enteric pathogens

\section{Microbiological features}

Gram negative BSI were the most frequent aetiological agents for all types of SOT. The ratio of gram positive to gram negative BSI was the highest in liver transplant recipients (Table 1). The most frequently isolated five pathogens were E. coli (28.7\%; $57.7 \%$ ESBL production), Klebsiella spp. (13.1\%; 48.5\% ESBL production), enterococci (13.5\%; 15\% VRE), coagulase negative staphylococci (CoNS) (11.6\%; $77.4 \%$ methicillin resistant) and $A$. baumannii $(7.3 \%, 72.2 \%$ MDR, 38.9\% possible XDR) (Table 2).

Antibiotic resistance among three enteric pathogens is shown in Figure 2. P. aeruginosa was isolated from 16 monomicrobial BSI episodes. Of these 16 isolates, 11 (68.8\%) were MDR; 9 (56.3\%) were possible XDR; 1 was possible PDR according to the proposed definitions for MDR, XDR and PDR (8). The susceptibilities of both levofloxacin and trimethoprimsulphamethoxazole among S. maltophilia isolates were $75 \%$. Methicillin resistance was detected in $22.2 \%$ (4/18) of S. aureus and $77.4 \%(24 / 31)$ of CoNS tested isolates. Methicillin resistance was higher among CoNS than S. aureus in our hospital.

There were $42(13.2 \%)$ polymicrobial BSI episodes which were distributed as follows: $16(16.3 \%)$ in the early period, 28 $(20.9 \%)$ in the mid-term, and $8(9.4 \%)$ in the late period after transplantation.

\section{Discussion}

Our hospital is one of the leading organ transplantation centres in Turkey. When we conducted a search using the keywords "solid organ transplantation", "infection" and "Turkey" in the medical electronic databases PubMed, Web of Science and EBSCO, we found only one retrospective study published from Turkey about BSI among SOT recipients (5). To the best of our knowledge, the conducted study was the first comprising both paediatric and adult recipients of three different types of transplantation from Turkey.

Post-transplant BSI was more common among liver recipients compared to other types of transplantation, which was statistically significant, especially in the early and the mid-term post-transplant period in the conducted study. Linares et al. (3) also yielded the same significance within 30 days posttransplantation. The incidence was higher among all types of SOT compared to the study performed by Moreno et al. (9) (10.4\% versus $7.3 \%$ in kidney recipients; $39.4 \%$ versus $10.4 \%$ in liver recipients; $18.8 \%$ versus $8.2 \%$ in heart recipients). Gram negative bacteria (61.1\%) were prominent among all types 


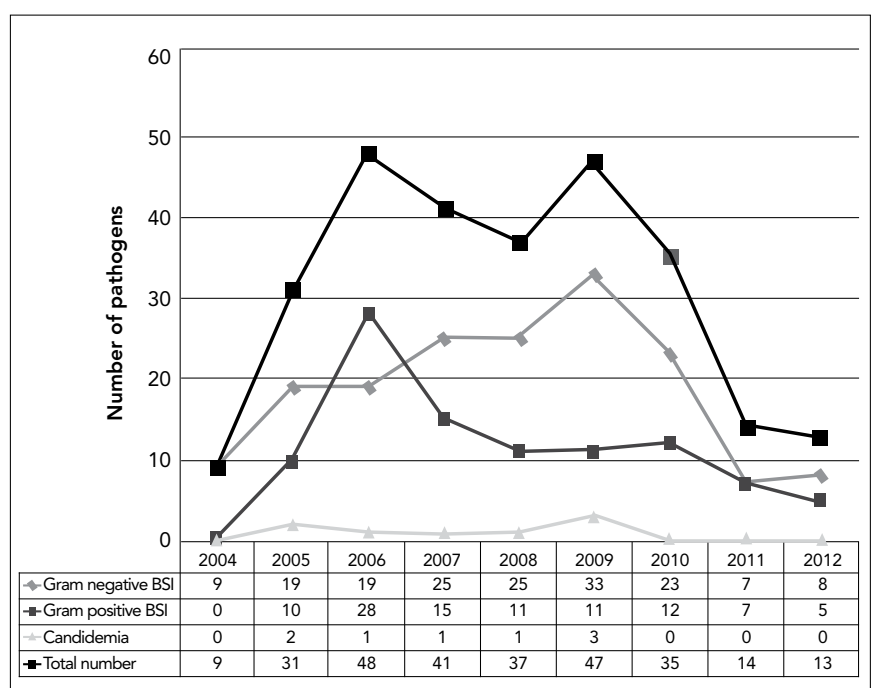

Figure 3. The distribution of pathogens of bloodstream infections by year

of SOT in our study in accordance with the previous studies (53\%-52.3\%) during the study period, except in $2006(1,10)$ (Figure 3). The study of Singh et al. (4) revealed a shift toward gram negative bacteria as the predominant pathogens in BSI among liver transplant recipients in their centre between 1989 and 2003. In contrast to this, national surveillance reports from Spain in the period between 2003 and 2005 revealed gram positive BSI to be predominant among SOT recipients, with the exception of kidney recipients (9). BSI among heart transplant recipients was published less often in the literature compared to BSI among other SOT types. Gram negative microorganisms predominated $(57.9 \%)$ in our centre among heart transplant recipients, which was in accordance with previous studies $(55.3 \%-57 \%)(11,12)$.

The most frequently isolated microorganism was $E$. coli from BSI among SOT recipients in our centre, of which $57.7 \%$ were ESBL-producing. ESBL production among E. coli and Klebsiella spp. isolates is also a large problem in Spain; nearly half of the isolates were reported to be ESBL positive (3). The percentage of ESBL production among $E$. coli isolates was $44.6 \%$ and $47.6 \%$ among $K$. pneumoniae isolates in Turkish intensive care units (ICU) (13). E. coli was the most common agent causing BSI in a previous study published from Mayo Clinic in the United States of America in which the prevalence of ESBL producing $E$. coli was $8 \%$ (14). Both the distribution of the causative microorganisms and the antibiotic resistance patterns change from country to country, and even from centre to centre. Each transplantation centre should do surveillance and interpret particular microbiology data in order to choose the appropriate antimicrobial prophylaxis and empirical therapy for SOT recipients.

In the present study, the resistance rates of Pseudomonas spp. against ceftazidime, ciprofloxacin and imipenem were $25 \%, 31 \%$ and $25 \%$, respectively. The Turkish National Nosocomial Surveillance Report revealed carbapenem resistance rate among $P$. aeruginosa isolates of ICU as $32.9 \%$ in 2011 (13). Shi et al. (15) reported resistance rates among Pseudomonas spp. against ceftazidime, ciprofloxacin and imipenem as $18 \%, 33 \%$ and $32 \%$, respectively, in China. The infections caused by multidrug-resistant, extensively drug-resistant and pan-drug resistant gram negative bacteria are a big problem all around the world. The antibiotic policy in the hospitals is an important part of infection control, in addition to isolation precautions. By definition, there is no drug of choice to treat infections caused by PDR microorganisms and this issue is worrisome for all healthcare centres.

BSI caused by Acinetobacter strains only susceptible to colistin, vancomycin-resistant enterococci and carbapenemresistant Enterobacteriaceae are emerging problems in our centre in the last five years in accordance with previous studies $(3,15,16)$. Carbapenem resistance in Enterobacteriaceae are due to OXA-48 type carbapenemases in Turkey. Fortunately, all Acinetobacter and carbapenem-resistant Enterobacteriaceae isolates were found to be susceptible to colistin in our institution. The reasons for the high numbers of drug-resistant strains are diverse. However, the widespread use of antibiotics both for prophylaxis and treatment in the pre- and posttransplant periods is of pivotal importance for the emergence of these resistant strains. Other factors probably associated with the emergence of antimicrobial resistant pathogens were reported as severity of underlying disease, cross-infection, post-transplant reoperation, post-transplant abdominal infection, and high dose immunosuppressive therapy for acute rejection (15).

The percentage of candidaemia after SOT in our centre (2.9\%) was similar to previous reports $(1.7 \%-7 \%)(1,6,10,11)$. The results of both the identification and antifungal susceptibilities of five Candida isolates yielded no fluconazole resistance. The most prominent species in candidaemia was $C$. albicans (80\%).

The number of living donor transplantation cases continues to be higher than deceased donor transplantation in our centre (687 versus 176) when compared with Western countries. Living donor transplantation seems to be the appropriate solution because of the shortage of deceased organ donation. Therefore, it was not a surprise for us to find a higher frequency of $\mathrm{BSI}$ episodes among recipients of both living kidney and liver transplantation (233/65 BSI episodes; 78.2\% versus $21.8 \%$ ). Nineteen BSI episodes were excluded from the evaluation as they were seen among heart transplantation recipients. Although they hypothesised a lower incidence, the study of Saner et al. (17) revealed a higher incidence in pulmonary and BSI compared with their deceased organ recipients' counterparts. Incidental comparison between the two groups is not suitable because this is a retrospective study. Well-designed, prospective, multicentre studies to compare infectious aetiology between living and deceased organ donation should be performed by researchers in Turkey where living donation is higher compared with Western countries.

The present study has some limitations. Firstly, the retrospective design which relies only on written data in medical charts and hospital computer system results in difficulties in the interpretation of patients. The second limitation was the underestimation of the incidence of BSI, as SOT recipients might have attended other centres for BSI treatment. We assume that the number should be small, as our patients are 
always willing to come to our institution, although they live far from our centre. Thirdly, we evaluated BSI, but unfortunately not the source of infection, outcome, or risk factors. The fourth limitation is reporting data only from one centre, which cannot be generalised for Turkey. Anyway, the results of this study were the first mainstay results from Turkey, which will probably be the standpoint for future research.

In conclusion, liver transplant recipients are more vulnerable to developing BSI than others. Gram negative BSI outnumber gram positive ones in all types of SOT. Carbapenem seems to be the first choice in empirical antimicrobial therapy in $\mathrm{BSI}$ among SOT recipients due to the high incidence of ESBL producing Enterobactericeae in Turkey. Possible XDR and possible PDR gram negative microorganisms including carbapanemase-producing Enterobacteriaceae will probably be the most prominent problem needing to be solved in the near future.

Ethics Committee Approval: This study was conducted with the approval of the Institutional Review Board and in accordance with the principles of the Helsinki Declaration.

Informed Consent: N/A.

Peer-review: Externally peer-reviewed.

Author contributions: Concept - Ö.K.A, H.A.; Design - A. Y., M.A.O.; Supervision - Ö.K.A., H.A.; Resource - A.A.; Materials - A.Y., A.A.; Data Collection\&/or Processing - M.H.D., A.A.; Analysis\&/or Interpretation - A.Y., M.A.O.; Literature Search - A.Y., M.H.D.; Writing -A.Y., Ö.K.A., M.H.D., M.A.O.; Critical Reviews - Ö.K.A., H.A.

Acknowledgements: We acknowledge the technical contributions of M. Özgür Sarı and Levent Altıntaş in editing this manuscript.

Conflict of Interest: No conflict of interest was declared by the authors.

Financial Disclosure: No financial disclosure was declared by the authors.

\section{References}

1. Candel FJ, Grima E, Matesanz M, Cervera C, Soto G, Almela M, et al. Bacteremia and septic shock after solid-organ transplantation. Transplant Proc 2005;37:4097-9. [CrossRef]

2. McClean K, Kneteman N, Taylor G. Comparative risk of bloodstream infection in organ transplant recipients. Infect Control Hosp Epidemiol 1994;15:582-4. [CrossRef]

3. Linares L, Garcia-Goez JF, Cervera C, Almela M, Sanclemente G,

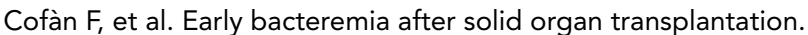
Transplant Proc 2009;41:2262-4. [CrossRef]

4. Singh N, Wagener MW, Obman A, Cacciarelli TV, de Vera ME, Gayowski T. Bacteremias in liver transplant recipients: shift to- ward gram-negative bacteria as predominant pathogens. Liver Transpl 2004;10:844-9. [CrossRef]

5. Serefhanoglu K, Timurkaynak F, Arslan H, Karakayalı H. Bacteremia among liver and kidney transplant recipients: etiological agents and antimicrobial susceptibilities. Braz J Infect Dis 2011;15:408-9. [CrossRef]

6. Silva M Jr, Marra AR, Pereira CA, Medina-Pestana JO, Camargo LF. Bloodstream infection after kidney transplantation: epidemiology, microbiology, associated risk factors, and outcome. Transplantation 2010;90:581-7. [CrossRef]

7. $\mathrm{CDC} / \mathrm{NHSN}$ Surveillance Definition of healthcare-associated infection and criteria for specific types of infections in the acute care setting. Available from: http://www.cdc.gov/nhsn/pdfs/ pscmanual/17pscnosinfdef_current.pdf

8. Magiorakos AP, Srinivasan A, Carey RB, Carmeli Y, Falagas ME, Giske CG, et al. Multidrug-resistant, extensively drug-resistant and pandrug-resistant bacteria: an international expert proposal for interim standard definitions for acquired resistance. Clin Microbiol Infect 2012;18:268-81. [CrossRef]

9. Moreno A, Cervera C, Gavalda J, Rovia M, de la Camara R, Jarque I, et al. Bloodstream infections among transplant recipients: results of a nationwide surveillance in Spain. Am J Transplant 2007;7:2579-86. [CrossRef]

10. Bert F, Larroque B, Paugam-Burtz C, Janny S, Durand F, Dondero $F$, et al. Microbial epidemiology and outcome of bloodstream infections in liver transplant recipients: an analysis of 259 episodes. Liver Transpl 2010;16:393-401.

11. Rodríguez $\mathrm{C}, \mathrm{Mu}-\mathrm{oz} \mathrm{P}$, Rodríguez-Créixems $\mathrm{M}$, Ya-ez JF, Palomo $J$, Bouza E. Bloodstream infections among heart transplant recipients. Transplantation 2006;81:384-91. [CrossRef]

12. Hsu RB, Chang $\mathrm{Cl}$, Fang $\mathrm{CT}$, Chang SC, Wang SS, Chu SH. Bloodstream infection in heart transplant recipients: 12-year experience at a university hospital in Taiwan. Eur J Cardiothorac Surg 2011;40:1362-7.

13. Ulusal Hastane Enfeksiyonları Sürveyans Ağı (UHESA) Raporu Özet Veri, 2011[Turkish]. Available from: http://www.rshm.gov.tr/ enfeksiyon/dosya/ybuinvaziv_2011.pdf

14. Al-Hasan MN, Razonable RR, Eckel-Passow JE, Baddour LM. Incidence rate and outcome of gram-negative bloodstream infection in solid organ transplant recipients. Am J Transplant 2009;9:835-43. [CrossRef]

15. Shi SH, Kong HS, Xu J, Zhang WJ, Jia CK, Wang WL, et al. Multidrug resistant gram-negative bacilli as predominant bacteremic pathogens in liver transplant recipients. Transpl Infect Dis 2009;11:405-12. [CrossRef]

16. Azap Ö, Otlu B, Yeşilkaya A, Yakupoğulları Y. Detection of OXA48-like carbapenemase-producing Klebsiella pneumoniae in a tertiary care center in Turkey: molecular characterization and epidemiology. Balkan Med J 2013;30:259-60. [CrossRef]

17. Saner FH, Olde Damink SWM, Pavlakovic G, van den Broek MAJ, Rath PM, Sotiropoulos GC, et al. Pulmonary and blood stream infections in adult living donor and cadaveric liver transplant patients. Transplantation 2008;85:1564-8. [CrossRef] 